\title{
Radiosensitizing effect of the histone acetyltransferase inhibitor anacardic acid on various mammalian cell lines
}

\author{
ROSEMARIE TEN CATE ${ }^{1}$, PRZEMEK KRAWCZYK ${ }^{2 *}$, JAN STAP $^{2 *}$, \\ JACOB A. ATEN ${ }^{2}$ and NICOLAAS A.P. FRANKEN ${ }^{1}$
}

${ }^{1}$ Laboratory for Experimental Oncology and Radiobiology (LEXOR), Center for Experimental Molecular Medicine, Department of Radiation Oncology, ${ }^{2}$ Department of Cell Biology and Histology, Academic Medical Center, University of Amsterdam, 1100 DE Amsterdam, The Netherlands

Received April 30, 2010; Accepted May 25, 2010

DOI: 10.3892/ol_00000134

\begin{abstract}
Agents that enhance the effectiveness of ionizing radiation have been investigated over many decades. A relatively new group of potential radiosensitizers consists of agents that inhibit histone acetyltransferases (HATs). This study evaluated the radiosensitizing properties of the HAT inhibitor anacardic acid (AA), used at a low-toxic concentration of $100 \mu \mathrm{M}$ in V79, SW1573 and U2OS cells. Radiation survival curves were analyzed according to the linear quadratic model. Significant radiosensitization by AA was only obtained in U2OS cells. AA significantly increased the value of the linear parameter $\alpha$, but not of the quadratic parameter $\beta$, indicating fixation of potentially lethal damage and an intact repair function of sublethal damage. The increase of the $\alpha$ value was also observed in SW1573 cells, but was not accompanied by a significant radiosensitization. A likely explanation for the enhancement of the $\alpha$ value may be an increase in the amount of lethal lesions due to the compacted chromatin structure. Despite the conflicting results of the radiosensitizing effect of AA in the three cell lines tested, the ability of AA to increase the $\alpha$ value suggests potential advantages for clinical application.
\end{abstract}

\section{Introduction}

Although radiotherapy has proven to be effective and is among the most common treatment modalities (1), much effort is being invested to further improve its effectiveness. Besides the development of new treatment techniques, the effects of

Correspondence to: Rosemarie ten Cate, Laboratory for Experimental Oncology and Radiobiology (LEXOR), Center for Experimental Molecular Medicine, Department of Radiation Oncology, P.O. Box 22700, 1100 DE Amsterdam, The Netherlands E-mail: r.tencate@amc.uva.nl

${ }^{*}$ Contributed equally

Key words: anacardic acid, radiosensitization, ionizing radiation, histone acetyltrasferase, histone acetyltrasferase inhibitor radiotherapy can be improved by concurrent administration of radiosensitizing agents. Previously, it was shown that chemotherapeutic agents such as cisplatin (cis-diamminodichloroplatinum II) and gemcitabine (difluorodeoxycytidine) are potent radioenhancers both in vitro and in vivo (2-4). Halogenated pyrimidines such as bromo-deoxyuridine (BrdU) and iodo-deoxyuridine (IdU) are also potent radiosensitizers (5-8). The aforementioned agents affect cells at the DNA level and are either directly incorporated into the DNA (BrdU, gemcitabine), form DNA adducts/crosslinks (cisplatin) or inhibit the production of deoxynucleotides, necessary building blocks for DNA syntheses and repair (gemcitabine).

Another group of potential radiosensitizers consists of agents that influence chromatin structure, thereby affecting DNA damage repair. Chromatin is a highly dynamic, yet efficiently organized structure that regulates almost all DNA-associated processes, including transcription, replication and repair (9). The basic chromatin unit is the nucleosome that consists of approximately 146 bp of DNA wrapped around a protein octamer containing two molecules each of histones H2A, H2B, H3 and H4 (10). The histone core contains highly conserved tail regions that can be covalently modified by phosphorylation, methylation and acetylation $(11,12)$. Histone acetylation is antagonistically regulated by histone acetylases (HATs) and histone deacetylases (HDACs). In general, histone acetylation is associated with a more open chromatin structure and active gene transcription, whereas deacetylation is associated with condensed chromatin and gene repression (12). Due to the intimate relationship between chromatin and DNA repair, disruption of the balance between HDACs and HATs may play a role in antineoplastic therapy. Subsequently, multiple HDAC inhibitors are currently undergoing clinical trials (13). HAT inhibitors have been less extensively studied, but may also possess anticancer potential.

Based on sequence and structure homology, three main classes of HATs have been described, including GCN5/PCAF, p300/CBP and the MYST family (MOZ, YBF2/SAS3, SAS2 and Tip60) (14). The exact functions that HATs play in cell biology have yet to be investigated in more detail, but it is clear that HATs play a role in gene transcription and that the effects of HATs can be both global and gene-specific. HATs are often part of large multiprotein complexes that determine 
their specificity. HATs primarily acetylate histones, but other proteins can be affected as well (15). The cell permeable salicylic acid analog anacardic acid (AA) is a potent, noncompetitive inhibitor of p300 and PCAF HAT activities (16). Sun et al (17) demonstrated that AA is an effective inhibitor of the MYST HAT Tip60. Tip60 was reported to play a role in the acetylation and activation of the ataxia telangiectasia mutant, an important mediator of DNA damage response (18). Ikura et al (19) reported that the inactivation of Tip60 resulted in a defective double strand break (DSB) repair. This defective repair may be due to the failure of Tip60 to induce local relaxation of chromatin at DSB sites, which may be necessary for proper DSB responses (9). This failure is supported by the finding that compact chromatin structure (heterochromatin) poses a (physical) barrier to the propagation of DSB signaling (20). Moreover, Biade et al (21) previously showed that chemicals that promote chromatin compaction were able to induce significant radiosensitization in tumour cells. Due to the influence of chromatin structure on DNA repair, HAT inhibitors are likely to posses radiosensitizing properties. Subsequently, the radiosensitizing effects of low concentrations of AA on a set of mammalian cell lines were studied.

\section{Materials and methods}

Cell lines. The human squamous lung carcinoma SW1573 cell line was cultured in Leibowitz-15 medium (Gibco-BRL Life Technologies, Breda, The Netherlands) supplemented with $10 \%$ heat-inactivated fetal bovine serum (FBS) and $2 \mathrm{mM}$ glutamine, $100 \mathrm{U} / \mathrm{ml}$ penicillin and $100 \mu \mathrm{g} / \mathrm{ml}$ streptomycin (Gibco). The human osteosarcoma U2OS cell line was cultured in Dulbecco's modified Eagle's medium (DMEM) (Gibco) supplemented with 10\% heat-inactivated FBS and $2 \mathrm{mM}$ glutamine, $100 \mathrm{U} / \mathrm{ml}$ penicillin and $100 \mu \mathrm{g} / \mathrm{ml}$ streptomycin. The Chinese hamster V79 lung fibroblasts were cultured in minimum essential medium (MEM) containing Hanks salts (Gibco) supplemented with $10 \%$ heat-inactivated FBS and $2 \mathrm{mM}$ glutamine, $100 \mathrm{U} / \mathrm{ml}$ penicillin and $100 \mu \mathrm{g} / \mathrm{ml}$ streptomycin. SW1573, U2OS and V79 cells were maintained in a $37^{\circ} \mathrm{C}$ humidified incubator with an air atmosphere of 0 , 10 and $2 \% \mathrm{CO}_{2}$, respectively. The cell lines were cultured as a monolayer in tissue culture flasks (Costar Europe Ltd., Badhoevedorp, The Netherlands) and passaged twice a week to ensure exponential growth.

Irradiation. Irradiation was performed with $\gamma$-rays from a ${ }^{137} \mathrm{Cs}$ source, yielding a dose rate of about $0.6 \mathrm{~Gy} / \mathrm{min}$. Cells were irradiated with doses of up to $8 \mathrm{~Gy}$.

Anacardic acid. A $100 \mathrm{mM}$ stock solution of AA (Calbiochem, cat no. 172050) was dissolved in dimethylsulfoxide (DMSO) just before use. For the experiments, AA was pre-diluted in medium at appropriate concentrations ranging from 50 to 300 $\mu \mathrm{M}$ and then added to the cells. AA concentrations of up to $300 \mu \mathrm{M}$ were used for the cytotoxicity measurement.

Clonogenic assay. Cytotoxicity of AA alone, ionizing radiation (IR) alone and combination treatment of AA and IR was measured using clonogenic survival assays (22). Cytotoxicity of AA alone was determined after a 4-h incubation at concen-
A V79 cells

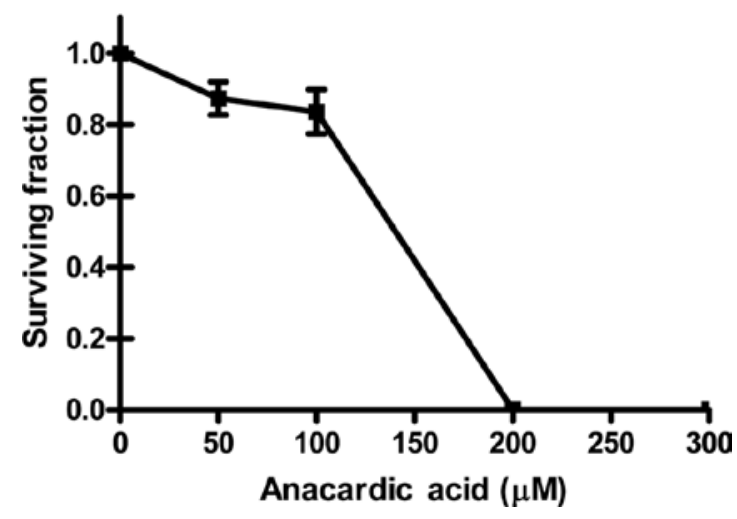

B SW1573 cells

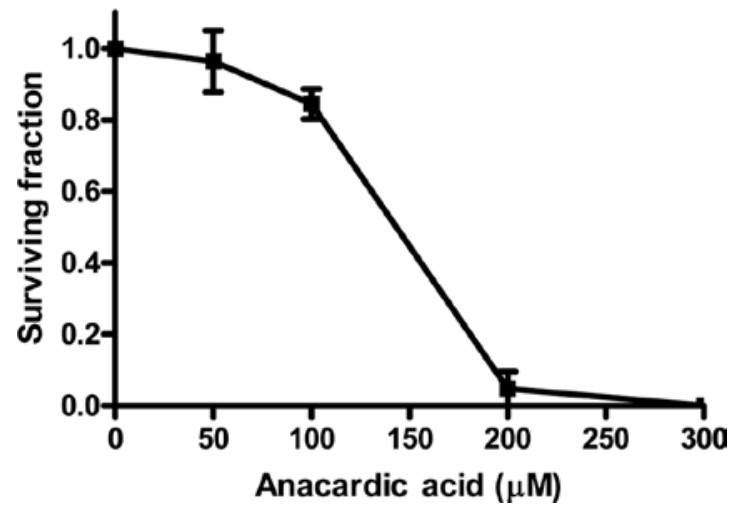

C U2OS cells

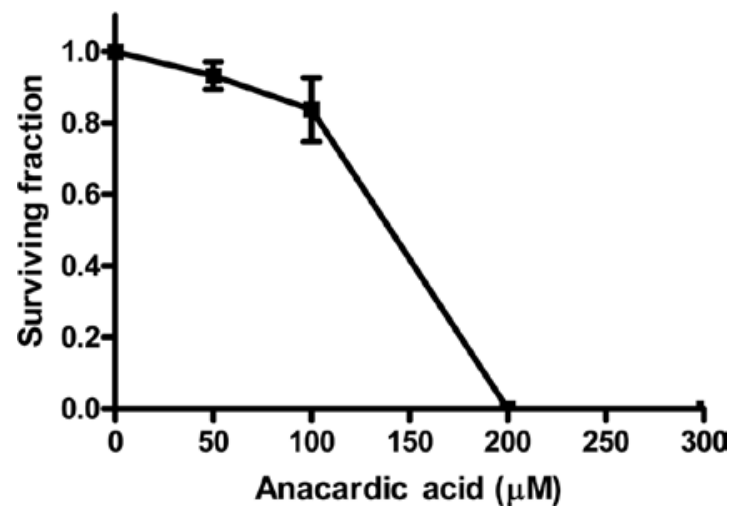

Figure 1. The cytotoxicity of anacardic acid (AA) in (A) V79, (B) SW1573 and (C) U2OS cells. Cells were treated with indicated doses of anacardic acid for $4 \mathrm{~h}$. Surviving fractions were calculated by normalization for the plating efficiency of DMSO-treated cells. Data points represent the average of 3 independent experiments \pm SEM.

trations of $0,50,100,200$ and $300 \mu \mathrm{M}$. Control experiments with DMSO were accomplished. Cells were plated in appropriate densities. After $3 \mathrm{~h}$, when cells were attached, they were treated with AA for $4 \mathrm{~h}$. In case of combined treatment, cells were irradiated with $0,2,4,6$ and 8 Gy $1 \mathrm{~h}$ after the onset of $100 \mu \mathrm{M} \mathrm{AA}$ incubation. Following treatment, the cells were washed twice with phosphate buffered saline, and fresh medium was added. Cells were allowed to form colonies for 10 days and were fixed and stained using a solution of $0.05 \%$ 
A
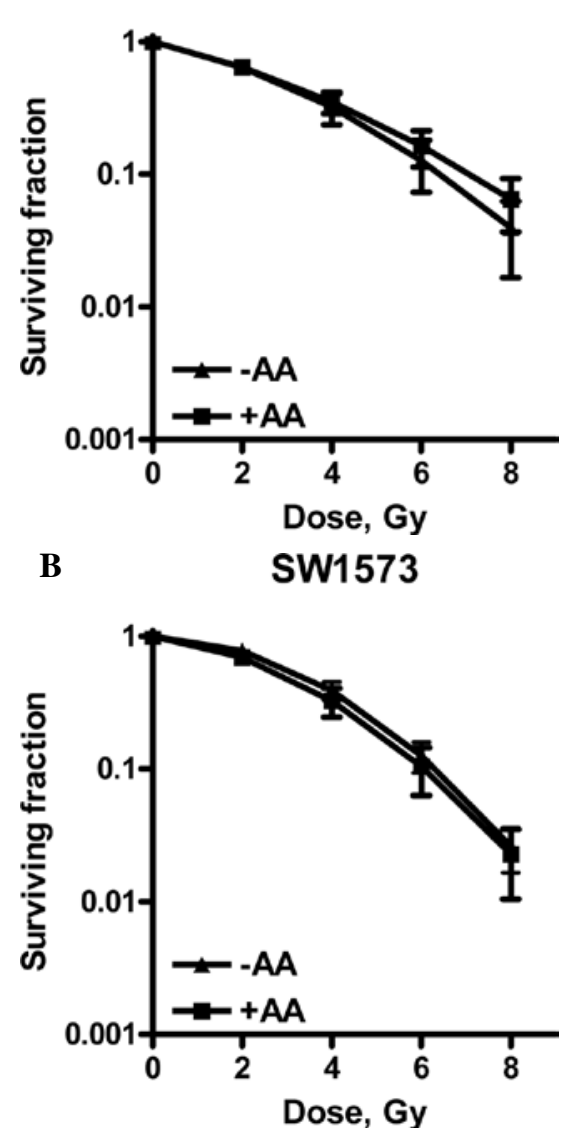

$\mathbf{C}$ U2OS cells

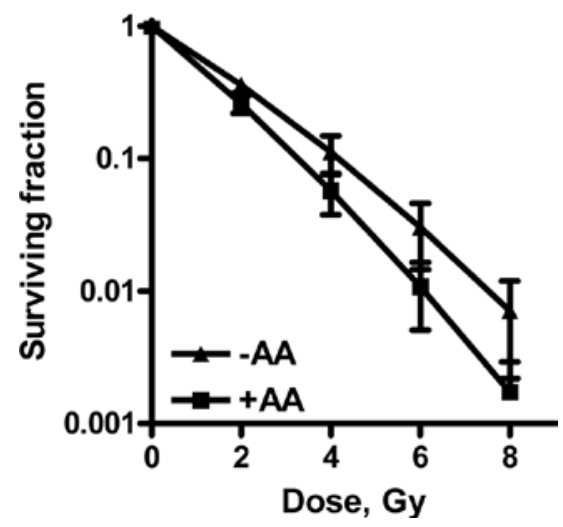

Figure 2. Radiation dose survival curves of (A) V79, (B) SW1573 and (C) U2OS cells after $4 \mathrm{~h}$ of treatment with $0.1 \%$ DMSO (ム) or after pre-treament with $100 \mu \mathrm{M}$ anacardic acid (AA, $\mathbf{m})$. Adherent cells were treated and irradiation was performed $1 \mathrm{~h}$ after the start of AA incubation. The medium was refreshed $3 \mathrm{~h}$ after irradiation. Surviving fractions are corrected for the toxicity of DMSO. Data points represent at least 3 experiments \pm SEM.

crystal violet in $6 \%$ glutaraldehyde. Colonies of $\geq 50$ cells were scored as originating from a single clonogenic cell (22).

Surviving fractions $(\mathrm{S}(\mathrm{D}) /(\mathrm{S}(0))$ after dose $\mathrm{D}$, corrected for the toxicity of AA alone $(S(0))$, were calculated and survival curves were analysed using SPSS statistical software (Chicago, IL, USA) by fitting the data using weighted, stratified, linear regression, according to the linear-quadratic formula: $S(D) / S(0)=\exp -(\alpha D+\beta D 2)(5,23,24)$. A procedure described by Franken et al (22) was used to test for a statisti-
Tabel I. Linear- quadratic parameters.

\begin{tabular}{lccc}
\hline & LQ parameter & $\alpha$, Gy-1 & $\beta$, Gy-2 \\
\hline V-79 & radiation only & $0.164 \pm 0.044$ & $0.030 \pm 0.008$ \\
& radiation + AA & $0.182 \pm 0.031$ & $0.020 \pm 0.005$ \\
SW1573 & radiation only & $0.015 \pm 0.026$ & $0.055 \pm 0.004$ \\
& radiation + AA & $0.089 \pm 0.041$ & $0.043 \pm 0.007$ \\
U2OS & radiation only & $0.475 \pm 0.050$ & $0.018 \pm 0.024$ \\
& radiation + AA & $0.635 \pm 0.065$ & $0.020 \pm 0.020$
\end{tabular}

$\alpha$ and $\beta$ values \pm SEM for V79, SW1573 and U2OS cells treated with DMSO $(0.1 \%)$ or anacardic acid (AA, $100 \mu \mathrm{M})$. Parameters were calculated from survival curves presented in Fig. 2; LQ, linear-quadratic.

cally significant difference between IR alone and the combined treatment of AA and IR curves.

\section{Results and Discussion}

To investigate the radiosensitizing properties of HAT inhibitors the commercially available AA was selected. To determine the appropriate dose for radiosensitization studies, cytotoxicity experiments were performed. Fig. 1 shows the cytotoxicity of AA for V79, SW1573 and U2OS cells incubated with concentrations up to $300 \mu \mathrm{M}$ for $4 \mathrm{~h}$.

It was noted that in the three cell lines the cytotoxicity of AA increases rapidly with concentrations above $100 \mu \mathrm{M}$. When incubated at a concentration of $200 \mu \mathrm{M}, \mathrm{V} 79$ and U2OS cell lines show no remaining viable clonogenic cells. A similar effect is achieved for SW1573 cells at a concentration of $300 \mu \mathrm{M}$. This effect indicates that the cytotoxicity of AA depends on the cell line tested. To evaluate the clinical applicability of AA as a radiosensitizer, a 4-h incubation at a concentration of $100 \mu \mathrm{M}$ was selected for further experiments. Treatment with this concentration was selected as it resulted in relatively low cytotoxicity in the examined cell lines showing a clonogenic survival $( \pm$ SEM) of $0.84 \pm 0.06,0.84 \pm 0.04$ and $0.84 \pm 0.09$ for V79, SW1573 and U2OS cells, respectively. These data were corrected for the toxicity of the AA solvent, DMSO.

To establish the radiosensitizing properties of AA, clonogenic assays were performed. Survival curves are shown in Fig. 2. Cells were plated in appropriate densities prior to the commencement of AA treatment and irradiation. In this experimental set-up, the cells were allowed to repair the radiation-induced DNA damage.

The survival curves were analysed according to the linearquadratic model (LQ) describing lethal events as a function of the dose (D) with the parameters $\alpha$ and $\beta$ as constants: $F(D)=$ $\alpha \mathrm{D}+\beta \mathrm{D}^{2}(5,23,24)$.

This model describes lethal events induced in the low-dose range $(\leq 2 \mathrm{~Gy} ; \alpha)$ separately from damage induced in the highdose range $(\geq 2 \mathrm{~Gy} ; \beta)$. In the low-dose range, damage is primarily induced by single-hit particle tracks, represented as lethal (LD) and potentially lethal damage (PLD). If not repaired, the latter type of damage becomes lethal, representing fixated (P)LD.

In the higher dose range LD is partly caused by the accumulation or interaction of multiple particle-induced sublethal 
damage (SLD) events. Table I shows the linear-quadratic parameters $\alpha$ and $\beta$.

Fig. 2 shows that U2OS cells are radiosensitized by incubation with $100 \mu \mathrm{M}$ AA for $4 \mathrm{~h}$, since the survival curves with and without AA showed a significant difference.

A significant increase of the $\alpha$ value by a factor of 1.3 was observed. The $\beta$ value was not significantly changed, indicating an intact repair of SLD (5). As repair function is unaffected, a likely explanation for the enhanced expression of LD induced by AA is due to an increase in the amount of directly lethal lesions.

One explanation for the increased number of lethal lesions induced by AA may be due to the hypoacetylated status and subsequent condensed structure of the chromatin. Biade et al (21) previously showed that compacted chromatin is a radiation hypersensitive target associated with the single-hit (low-dose) killing of tumour cells.

In contrast to U2OS cells, neither V79 nor SW1573 cells were radiosensitized by incubation with $100 \mu \mathrm{M}$ AA. SW1573 cells showed a significant increase of the $\alpha$ value by a factor of 5.9. This increase was accompanied, however, by a decrease of the $\beta$ value by a factor of 0.78 , indicating the enhanced repair of SLD. This decrease explains why the complete survival curves with and without AA were not significantly different for SW1573 cells.

Few studies have been published regarding the effect of AA on radiosensitization. In contrast to our results, Sun et al (17) found a distinct radiosensitizing effect of a 4-h and $40 \mathrm{~min}$ incubation in the presence of $30 \mu \mathrm{M}$ AA in HeLa, SCC35 and SQ20B cells. These authors suggested that the radiosensitizing properties of AA may be due to the inhibition of the Tip60-dependent activation of ataxia telangiectasia mutant and DNA-PKcs protein kinases, proteins essential in cellular responses to DNA damage.

The hypoacetylation of $\mathrm{H} 4$ histones and a moderate increase in sensitivity to ionizing radiation by the HAT inhibitors copper sulfate $\left(\mathrm{CuSO}_{4}\right)$ and nickel chloride $\left(\mathrm{NiCl}_{2}\right)$ was demonstrated by Song et al (25) when using non-toxic concentrations on yeast cells.

Bandyopadhyay et al (26) reported an ultraviolet-sensitizing effect in the human lung $\mathrm{H} 358$ cancer cell line when using the bisubstrate adduct spermidine-CO- $\mathrm{CH}_{2}-\mathrm{CoA}\left(\mathrm{Spd}\left(\mathrm{N}^{1}\right)-\mathrm{CoA}\right)$. The radiosensitizing effect correlated with an increased inhibition of histone acetylation and was accompanied by a transient arrest of DNA synthesis, transient delay in S-phase progression and the inhibition of nucleotide excision repair and DNA double strand break repair.

Although we did not find radiosensitization in all of the cell lines studied, the likelihood of AA to increase $\alpha$ suggests potential advantages for clinical application, especially for low-dose fractionated radiotherapy. However, further intensive studies extended to other cell lines are required to elucidate whether radiosensitization is due to an increased (P)LD and/or the effects on DNA repair. Insight into the mechanism of radiosensitization is required if it is to be used in a therapeutic setting.

\section{Acknowledgements}

The authors would like to thank Professor G.W. Barendsen for his critical comments and useful suggestions on the manuscript. The Maurits and Anna de Kock and the Nijbakker
Morra foundations are acknowledged for sponsoring laboratory equipment.

\section{References}

1. Joiner M, van der Kogel A and Steel G: Introduction: the significance of radiobiology and radiotherapy for cancer treatment. In: Basic Clinical Radiobiology. 4th edition. Joiner and van der Kogel A (eds). University Press/Hodder Arnold, Oxford: pp1-10, 2009.

2. Haveman J, Castro Kreder N, Rodermond HM, Franken NA, Stalpers LJ, Zdzienicka MZ and Peters GJ: Cellular response of X-ray sensitive hamster mutant cell lines to gemcitabine, cisplatin and 5-fluorouracil. Oncol Rep 12: 187-192, 2004.

3. Bergs JWJ, Franken NA, ten Cate R, van Bree C and Haveman J: Effect of cisplatin and gamma irradiation on cell survival, the induction of chromosomal aberrations and apoptosis in SW-1573 cells. Mutat Res 594: 148-154, 2006.

4. Bergs JWJ, Franken NA, Haveman J, Geijsen ED, Crezee J and van Bree C: Hyperthermia, cisplatin and radiation trimodality treatment: a promising cancer treatment? A review from preclinical studies to clinical application. Int J Hyperthermia 23: 329-341, 2007.

5. Franken NA, van Bree C, Kipp JBA and Barendsen GW: Modification of potentially lethal damage in irradiated Chinese hamster V79 cells after incorporation of halogenated pyrimidines. Int J Radiat Biol 72: 101-109, 1997.

6. Franken NA, van Bree C, Veltmaat MAT, Rodermond HM, Haveman J and Barendsen GW: Radiosensitization by bromodeoxyuridine and hyperthermia: Analysis of linear and quadratic parameters of radiation survival curves of two human tumor cell lines. J Radiat Res 42: 179-190, 2001.

7. Iliakes G, Pantelias and Okayasu R: Mechanism of radiosensitization by halogenated pyrimidines: effect of BrdU on radiation induction of DNA and chromosome damage and its correlation with cell killing. Radiat Res 119: 286-304, 1989.

8. Franken NA, van Bree C, Veltmaat MA, Ludwików G, Kipp JB and Barendsen GW: Increased chromosome exchange frequencies in iodo-deoxyuridine-sensitized human SW-1573 cells after $\gamma$-irradiation. Oncol Rep 6: 59-63, 1999.

9. Squatrito M, Gorrini C and Amati B: Tip60 in DNA damage response and growth control: many tricks in one HAT. Trends Cell Biol 16: 433-442, 2006.

10. Luger K, Mäder AW, Richmond RK, Sargent DF and Richmond TJ: Crystal structure of the nucleosome core particle at 2.8 Å resolution. Nature 389: 251-260, 1997.

11. Tony Kouzarides: Chromatin modifications and their function. Cell 128: 693-705, 2007.

12. Shahbazian MD and Grunstein M: Functions of site-specific histone acetylation and deacetylation. Annu Rev Biochem 76: 75-100, 2007.

13. Kristensen LS, Nielsen HM and Hansen LL: Epigenetics and cancer treatment. Eur J Pharmacol 625: 131-142, 2009.

14. Lee KK and Workman JL: Histone acetyltransferase complexes: one size doesn't fit all. Nat Rev Mol Cell Biol 8: 284-295, 2007.

15. Dekker FJ and Haisma HJ: Histone acetyl transferases as emerging drug targets. Drug Discov Today 14: 942-948, 2009.

16. Balasubramanyam K, Swaminathan V, Ranganathan A and Kundu TK: Small molecule modulators of histone acetyltransferase p300. J Biol Chem 278: 19134-19140, 2003.

17. Sun Y, Jiang $X$, Chen $S$ and Price BD: Inhibition of histone acetyltransferase activity by anacardic acid sensitizes tumor cells to ionizing radiation. FEBS Lett 580: 4353-4356, 2006.

18. Sun Y, Jiang X, Chen S, Fernandes N and Price BD: A role for the Tip60 histone acetyltransferase in the acetylation and activation of ATM. Proc Natl Acad Sci 102: 13182-13187, 2005.

19. Ikura T, Ogryzko VV, Grigoriev M, Groisman R, Wang J, Horikoshi M, Scully R, Qin J and Nakatani Y: Involvement of the Tip60 histone acetylase complex in DNA repair and apoptosis. Cell 102: 463-473, 2000.

20. Goodarzi AA, Noon AT and Jeggo PA: The impact of heterochromatin on DSB repair. Biochem Soc Trans 37: 569-576, 2009.

21. Biade S, Stobbe CC, Boyd JT and Chapman JD: Chemical agents that promote chromatin compaction radiosensitize tumour cells. Int J Radiat Biol 77: 1033-1042, 2001. 
22. Franken NA, Rodermond HR, Stap J, Haveman J and van Bree C: Clonogenic assay of cells in vitro. Nat Protoc 1: 2315-2319, 2006.

23. Barendsen GW: Parameters of linear-quadratic radiation doseeffect relationships: dependence on LET and mechanisms of reproductive cell death. Int J Radiat Biol 71: 649-655, 1997.

24. Barendsen GW, van Bree C and Franken NA: Importance of cell proliferative state and potentially lethal damage repair on radiation effectiveness: implications for combined tumor treatments. Int J Oncol 19: 247-256, 2001.
25. Song S, McCann KE and Brown JM: Radiosensitization of yeast cells by inhibition of histone H4 acetylation. Radiat Res 170: 618-627, 2008

26. Bandyopadhyay K, Banères JL, Martin A, Blonski C, Parello J and Gjerset RA: Spermidinyl-CoA-based HAT inhibitors block DNA repair and provide cancer-specific chemo- and radiosensitization. Cell Cycle 8: 2779-2788, 2009. 